\title{
The new millennium Ethics of housing technology
}

\author{
S. Mahmoud Issa \& S. Ezzeldeen \\ Department of Architectural Engineering, Department of Basic Sciences, \\ Menofia University, Egypt
}

\begin{abstract}
Before the turn of the century, information technology was spreading worldwide knowledge regarding global environmental changes and their expected implications on the health of the globe's inhabitants. New approaches to deal with these hazards were initiated, including the technological approach in which Ethics stresses human values, human responsibilities and the internal human aspects of our global community.

From an ethical point of view, human conduct and the actions of professionals, especially in the case of ozone depletion, should lead to the revival of nature, the prevention of pollution and the application of sustainability as far as development and production are concerned. This conforms to the principles of the Engineers' code of Ethics as set by ABET. As Housing Technology provides the indoor environment that accommodates inhabitants, this paper will investigate, through an analytical approach, the Ethics that should be applied in providing peoples' needs, given the environmental changes and sustainability concepts in development, production and housing.

The study will include: housing technology functions, indoor housing pollution, sick building syndrome, the role of technology in protecting human rights and the code of practice for professionals and the ethical principles it formulates.

Keywords: global environmental changes, housing technology, indoor pollution, sick building syndrome, ethics, Engineers' code of Ethics.
\end{abstract}




\section{Introduction}

Last century's technology advancement, known as Information Technology, paved the way for closer relations and faster communications between the corners of the globe.

By spreading the news that the "World Meteorological Organization observed a $45 \%$ depletion of the ozone layer over one-third of the northern hemisphere, from Greenland to western Siberia, for several days during the winter of 1995-1996", it became clear that humanity has a pressing need to face the limited nature of its planet and should address the issues related to coping with the impact of environmental hazards to human health. Keeping pressure on the system within manageable limits necessitates various approaches to the problem that should have for the basis for an ethical directive and a distribution of responsibilities for the causes and effects of all the emissions that lead to environmental changes and ozone depletion.

\section{Environmental hazards: causes and effects}

There has been an increased recognition that environmental hazards to human health include climate change, stratospheric ozone depletion, loss of biodiversity as well as changes in hydrological systems and supplies of freshwater, land degradation and stress on food-producing systems [1]. Human activities, such as the burning of fossil fuels, resulted in the altering of the chemical composition of the atmosphere through the build-up of greenhouse gases and the release of atmospheric pollutants (such as chlorofluorocarbons), which deplete the lower ozone layer and increases human exposure to ultraviolet radiation, causing skin cancer and cataracts. Appreciation of this scale and of the type of influence on human health requires a new perspective, which focuses on ecosystems and on the recognition that the foundations of long-term good health in populations rely in great part on the continued stability and function of the biosphere's lifesupporting systems. It also brings an appreciation of the complexity of the systems upon which we depend. And that led to the formulation of the "Montreal Protocol, which acts to reduce emissions of pollutants that weaken the ozone layer. Although this international agreement is proving highly effective in reducing risks in the long term, Ultra Violet radiation remains a health hazard" [1].

\subsection{Environmental pollutants}

Ozone is composed of three Oxygen atoms, and is unstable [2]. The formation of Ozone has been taking place for many millions of years and its stability is attributed to Nitrogen compounds in the atmosphere. Ozone forms in the atmosphere by the action of sunlight on oxygen. Ozone higher up in the atmosphere protects life on Earth [2]. Ozone lower in the atmosphere (troposphere) can be toxic and a pollutant. Chlorofluorocarbons (CFCs) were developed in the 1930s. Their primary uses were for industrial, commercial, and 
household appliances. CFCs are non-toxic, non-flammable, and non-reactive with other compounds, and were first invented to replace dangerous sulphur dioxide and ammonia refrigerants. Most CFCs do not dissolve in water and therefore reach the atmosphere [2]. When released into the atmosphere, CFCs rise and are broken down by sunlight. Chlorine molecules interact with Ozone and destroy it.

\subsubsection{Indoor pollutants}

Studies have shown that the levels of some pollutants can reach high levels indoor, which was not previously known. Well-sealed buildings present protection (to some extent) from air pollution, depending on the type of pollutant. The following lists, some of the pollutants relate to air tight, sealed buildings and which are harmful to human beings:

Asbestos: It is now known that asbestos is a dangerous air pollutant. Asbestos is a fiber material, which has fine hair particles suspended in the air that cause lot of health problems if inhaled into to the lungs. Exposure to this substance for long periods of time results in a chronic disease known as asbestosis. This happens to people living around asbestos mines and near factories using this substance. Building materials containing friable (that can be crushed by hand) asbestos have a significant effect on pollution. Asbestos uses include fireresistant materials as well as heat and sound insulation. It could also be used as a reinforcement material in cement pipes and in floors and ceiling products.

Formaldehyde: Formaldehyde exists in the compressed wood used in furniture and on walls, in medium density fiberboard and in insulating foam manufactured from urea-formaldehyde. The latter is chemically unstable, and can release free formaldehyde from the volatile formaldehyde that did not react and exists in the resin, and from the hydrolysis of the resins itself. The formaldehyde that does not react is responsible for the high level of formaldehyde indoors.

Volatile Organic compounds (VOCs): This is a category that contains a very large number of easily evaporated organic compounds. It includes hazardous air pollutants. To varying degrees, VOCs contribute to the formation of ground level photochemical ozone.

There are many sources of VOCs. Motor vehicle exhausts are a major and pervasive source, in addition to combustion engines that burn hydrocarbons inefficiently. There are also small sources of VOCs, including wood-paintings and organic solvent-based paints, freshly painted houses (chlordane and asbestos) [3], and mercury, chlordane and asbestos resulting from paintings and the use of acoustical tiles. Additionally, there are some building materials like gypsum, and places such as auto maintenance shops and bakeries, which emit large amounts of ethanol, contributing to smog (visible air pollution) formation [4]. Natural VOC emissions are not a problem by themselves but must be figured 
into a strategy to reduce urban ozone formation [5]. VOCs contribute to health problems when sensitive individuals may react to those emissions with attacks of asthma, or other respiratory problems.

Measures to reduce those emissions in building materials implies the use of alternative paints including natural, hypoallergenic and low-biocide paints that have been developed to reduce those emissions [4]. The process of ventilation and storing gypsum boards separately could be applied to prevent absorption of these compounds by other surfaces or materials in the building's interior, as well as the use of recycled gypsum that would be free of volative organic compounds [4].

Radon: Radon is a radioactive material. In general, radioactive pollution results from natural sources or from human activity. Natural sources of radiation are radioactive gases that come out of the soil and rocks. Radiation caused by human activity arises when using weapons and in nuclear reactors used for the generation of energy and from the disposal of waste from these reactors.

The major source of radon comes from the soil, which constitutes $90 \%$ of the radon level in buildings. Other sources come from well water, natural gas and building materials.

\section{Housing technology: functions, concerns and impacts}

Acquiring adequate housing is a major issue for many individuals. The problems encountered to find a place to live that meets one's needs whilst still being affordable and in the preferred location vary according to one's ability and physical condition. This applies in the first place to elderly and disabled people whose additional problems include lack of accessibility and sometimes discriminatory attitudes in the place they have chosen to live.

Through technology and advances in design, housing needs should be satisfied without presenting risks or hazards to the users. Knowledge of innovative ways to build houses that can accommodate residents and visitors with disabilities has been available to professionals with the help of Assistive Technology i.e. as defined in the US "the broad range of devices, services, strategies and practices that are designed to increase the functional competencies of persons with disabilities" [6]. These include, but are not limited to, environmental control systems or "devices designed to permit the user to exercise greater control over devices and functions within their immediate vicinity" [6]. The control systems that the technology provides include: fire alarms, automatic control of entrances and of lighting at night in addition to the systems alarm for burglars at night and the simulation presence for maximal safety and security. That, in addition to processing or setting comfortable temperature through a system of "Automatic regulation of lighting and heating" [7]. The system also includes the use of Remote Control for service functions in order to achieve maximal comfort [7].

Advances in housing technology have led to refined methods of housing construction, which incorporate the advantages of materials testing with the 
process of manufacturing the relevant parts in innovative systems. Newly-tested materials could provide structural superiority, improved thermal dynamics, no rotting and protection from pest problems. While conventional building methods and materials have registered incremental changes across time (the major choice for providing the close to nature internal environment) there have been certain issues which the housing industry, the second largest industry worldwide after the food industry, still considers of greater importance than adapting new techniques or using different innovative materials. These include, amongst others, the internal indoor environment and its effect on people's health and on their ability to perform their activities, given the use of highly effective insulation materials, as well as the sustainability of these products for future generations. While innovations and improvements have brought tremendous changes in housing quality, the use of building materials preventing toxic or harmful effects on its users is still a major concern for the housing industry.

"The selection of building materials greatly impacts the sustainability of a project. By choosing building materials wisely, such as considering the complete life cycle of the materials, a designer and/or builder can reduce the impact of the project on the environment. Careful material selection can minimize the depletion of resources, including raw materials, such as wood and metals, as well as energy and water used in the manufacturing process. It also can allow for efficient reuse or recycling of materials and building components if a building or facility is to be deconstructed or demolished. Finally, building materials choices may alleviate environmental impacts created by the manufacturing process, as well as indoor air quality in the final structure" [8].

"Analyzing the environmental impacts of building materials can be a difficult process". A life-cycle analysis, which accounts for the impacts of resource extraction through manufacturing, use, and disposal, involves extensive data collection and analysis and explains the impact of the energy used to do these needed processes. Some of this analysis has already been conducted and results are available through various lists and databases of environmentally preferable building products [8].

\subsection{Environmentally preferable materials}

The federal government of the USA defines environmentally preferable products (EPP) as goods that have a lesser or reduced effect on human health and the environment when compared to competing products that serve the same purpose. Environmentally preferable attributes include reduced toxicity, the use of recycled materials, and increased energy efficiency.

Building for Environment and Economic Sustainability (BEES) developed BEES software that measures the environmental performance of building products using an environmental life cycle approach. Twelve environmental impacts are now included in each product's environmental performance score: global warming, acidification, fossil fuel depletion, indoor air quality, habitat alteration, ozone depletion, water intake, criteria air pollutants, smog, ecological toxicity, and human health [9]. 
All the finishes and materials considered to be "Environmentally Preferable" have been formulated and manufactured with children, the elderly, and the chemically sensitive in mind, and were recommended for people with allergies, with sick building syndrome or multiple chemical sensitivity.

\subsubsection{Multiple Chemical Sensitivity (MCS)}

"Multiple Chemical Sensitivity (MCS) is the name given to the broad issue of reactions to specific or cumulative chemicals in the environment. One of the difficulties in classifying MCS as an illness has been the complex nature of chemicals in the environment and the interaction effects with and within the human body. The length of exposure, the concentration of the chemical(s), and the individual's threshold of resistance are also factors complicating a simple definition. In the relatively few but growing documented cases of severe reactions to chemicals, there seems to be no single stimuli or predictor of reactions" [10].

Table 1: $\quad$ Peoples' chemical sensitivity.

\begin{tabular}{|l|l|l|}
\hline \multicolumn{1}{|c|}{ Chemical } & \multicolumn{1}{|c|}{ Where found } & \multicolumn{1}{|c|}{ Reactions } \\
\hline Formaldehyde & $\begin{array}{l}\text { carpet, plywood, particle board } \\
\text { insulation, adhesives }\end{array}$ & $\begin{array}{l}\text { sore throat } \\
\text { headaches }\end{array}$ \\
\hline Pesticides & $\begin{array}{l}\text { bug sprays, lawn chemicals, } \\
\text { household cleaners }\end{array}$ & $\begin{array}{l}\text { skin/eye/respiratory } \\
\text { digestive tract/nervous } \\
\text { system }\end{array}$ \\
\hline Nitrogen dioxide & $\begin{array}{l}\text { Unvented gas stoves/heaters } \\
\text { Carbon monoxide gas } \\
\text { appliances }\end{array}$ & $\begin{array}{l}\text { respiratory problems } \\
\text { headaches/dizziness }\end{array}$ \\
\hline Solvents & $\begin{array}{l}\text { household cleansers, } \\
\text { paints strippers, gasoline }\end{array}$ & $\begin{array}{l}\text { respiratory problems } \\
\text { fatigue/dizziness }\end{array}$ \\
\hline Latex & paints, gloves, caulking & allergic reactions \\
\hline $\begin{array}{l}\text { Dyes (especially } \\
\text { dark blue) }\end{array}$ & $\begin{array}{l}\text { clothing, curtain, tablecloths, } \\
\text { napkins, rags, furniture }\end{array}$ & skin reaction \\
\hline
\end{tabular}

The previous table summarizes some chemicals to which people are most likely to have sensitivity.

\subsubsection{Sick Building Syndrome (SBS)}

Sick Building Syndrome "is a combination of ailments (a syndrome) associated with an individual's place of work (typically, but not always, an office building), although there have also been instances of SBS in residential buildings. A 1984 World Health Organization report into the syndrome suggested up to $30 \%$ of new and remodeled buildings worldwide may be linked to symptoms of SBS" [11]. 
Building occupants complain of symptoms such as: a) Headaches b) Eye, nose, or throat irritation c) Dry cough; dry or itchy skin d) Dizziness and nausea e) Difficulty concentrating f) Fatigue j) Sensitivity to odors.

For SBS to exist, these symptoms must disappear soon after the occupants go outside. The contributing factors often relate to the design of the built environment, and may include combinations of some or all of the following: a) indoor air pollution; b) artificial fragrance, such as dryer sheets c) limited access to natural sunlight; d) poor ventilation or heating; e) bad acoustics; f) poorly designed furniture; g) poor ergonomics; h) chemical contamination; i) biological contamination.

The solution to this problem lies in the following activities: pollutant source removal or modification: maintenance of "Heat Ventilation and Air conditioning HVAC" systems, replacement of water-stained ceiling tiles and carpeting, institution of smoking restrictions, venting containment source emissions to the outdoor storage and use of paints, adhesives, solvents, and pesticides in well ventilated areas, and use of these pollutant sources during periods of nonoccupancy.

\section{Indoor pollution abatement measures}

The following measures are undertaken to reduce the impact of air pollutants inside buildings [12].

\subsection{Choosing the appropriate building specification}

\subsubsection{Exclude the source of pollution}

In newly-built houses or renovated ones the choice of building materials should exclude materials emitting pollutants, such as formaldehyde and medium density fibreboard, and use alternatives such as solid wood and decorative gypsum board. Covering floors with composite materials should be avoided and instead ceramic or wood should be used [13]. Air pollution by aliphatic and aromatic hydrocarbons and also by chlorinated hydrocarbons can be prevented by selecting the right paint finishes and furnishings. An example is the water-based paint that emits lower levels of volatile organic compounds than oil-based paints and varnishes.

\subsubsection{Removal of the polluting source}

This is an effective way to the abatement of formaldehyde. Asbestos is removed when it causes a pollution problem because it is used in some developing countries, although it is now forbidden due to the dangerous impact it has on health.

\subsubsection{Choosing the building location}

Air pollution due to radon can be avoided indoors by choosing the location of the building. The single most important factor, which enables radon to enter a building, is through the soil. For example, sand soil of high permeability constitutes a good medium for radon transfer, in contrast to muddy soil, which decreases radon penetration. 


\subsubsection{Climate control}

Climate control can be used to decrease indoor pollutant levels. For example the levels of formaldehyde indoors are influenced greatly by the temperature and the relative humidity. This is done by decreasing the source of emission. Taking the science of chemical kinetics into consideration, we can find that temperature is the single most important factor in emitting volatile organic compounds from several construction materials and from some paints.

\subsubsection{Source treatment}

Modifications can be made to decrease the emission that causes the pollution. For example, materials containing asbestos can be sprayed by an appropriate material to achieve this goal. The cost of this operation is about $50 \%$ of the cost of removing asbestos.

\subsubsection{Ventilation}

Ventilation is used to decrease indoor pollution. This decreases the results from dilution and from the removal of pollutants. Methods involved include infiltration and exfiltration and natural ventilation, in addition to forced or mechanical ventilation. The first two methods constitute the methods for exchange of air in buildings. Forced ventilation and to some extent infiltration and exfiltration constitute the major methods by which air is exchanged in large offices and multi-storey buildings.

\subsubsection{Air purification}

Air purification or air cleaning is a procedure similar to ventilation since both involve decreasing the air pollutants. Air dust purification systems are more efficient than those used for gaseous pollutants. The principles of collecting dust are applied to a variety of particles regardless of their source, which is different to gaseous pollutants. Common techniques for collecting dust in the air are filtration and electrostatic precipitation.

\section{Codes of engineering ethics}

As is the case for other professions, engineers of different specializations attempted to formulate rules or standards of conduct in the form of codes of ethics [14]. Engineers in the USA accomplished this code, while others did not have the same opportunity and the situation has been left to one's conscience and to regulations set by the authorities. These codes protect the public and preserve the dignity of the profession. There is no single code of ethics for all engineering specializations [15]. However, they all agree upon certain issues as to what is known as ethical behavior. Therefore, there is a similarity among the codes formulated. One of the most recognized is the code of ethics published by the Accreditation Board for Engineering and Technology (ABET) [16]. The code consists of four fundamental principles and seven fundamental canons or authoritative rules. The fundamental principles state that engineers "uphold and advance the integrity, honour and dignity of the engineering profession by the 
following: using their knowledge and skill for the enhancement of human welfare, being honest and impartial and serving with fidelity the public, their employers and clients, striving to increase the competence and prestige of the engineering profession, and supporting the professional and technical societies of their disciplines". These principles and canons constitute the basic rules of professional behavior, which can be applied to different scientific specialties such as the codes of ethics of chemists [17].

Since undertaking ethical decisions is sometimes difficult, more detailed guidelines have been prepared in order to be used with the fundamental canons of ethics.

\section{Conclusion}

The new millennium has been the beginning of a new era marked with new approaches for dealing with all the environmental problems that characterized the last century and resulted in damage to our planet from as a result of human activities.

While Information Technology has brought the awareness of the threats to human health to our attention, housing technology that provides the environment accommodating its users should fulfill its needs without endangering their health or depriving them from their rights to safety and security. The choice of building materials should be based on careful investigation of their properties, efficiency for reuse and impact and effects on people and the surrounding environment.

The role of housing technology's in allowing disabled and elderly people to benefit from the available assistive technology should be enforced as this allows the members of this group to reside in their own homes and participate in a social life.

As ethics is a major factor affecting professionals' conduct, which in turn reflects on other society members, there is a need for it to be enforced through the following recommendations:

1) Each profession should have a code of ethics to abide by in every country.

2) People all over the world should be encouraged to exchange their views and experiences in the field of ethics.

3) Examples of engineers who made good contributions to their own profession and followed the code of ethics that was formulated or even the common rules of ethics, if it has not been formulated, should be made known to other members of the profession.

4) Knowledge should be made available to everyone.

The following recommendations are presented for the housing sector:

1) The professional level of architects and engineers, such as chemical and civil engineers, should be raised, through awards for the best performance and ethical conduct.

2) Architects and engineers (such as the chemical and civil) should be encouraged to be aware of the latest innovations in the field and to experiment for new safer materials. 


\section{References}

[1] WHO, Global environmental Change, http://www.who.int/globalchange/ en/ 2006

[2] Michigan University, Ozone depletion http://www-personal.umich.edu/ 2006.

[3] Hewehy, M., "Air Quality in Work Environment (Case Study) in Office Buildings in Egypt", CDC $28^{\text {th }}$ Annual Seminar, Cairo, December 1999.

[4] AIA - American Institute of Architects (2001), "Environment Resource Guide", at www.sustainableabc.com/sustaindev.html. 2004.

[5] Hill, M., Understanding Environmental Pollution" Cambridge Press UK.

[6] Allen, B. and Dillon, B., Environmental Control and Field Bus systems: A Study of field bus systems and their potential environmental control application for people with disabilities, Central Remedial Clinic, Dublin Ireland.

[7] Dewsbury, G., "Smart Thinking: Some thoughts on Designing a Smart House" available on line from www.gdewsbary.uykideas.com//smarthouse. $\underline{\mathrm{html}} 2003$

[8] Navy WBDG “Green Product” Resource Page, http://p2library.nfesc. navy.mil/P2_Opportunity_Handbook/13_7.html

[9] Minnesota Office of Environmental Assistance, Sustainable Bldg Products http://www.buildinggreen.com/auth/article.cfm?fileName=040101a.xml

[10] Ohio State Univ. Fact Sheet, //www. ohioline.osu.edu/cd-fact/0192.html

[11] Godish, T., Air Quality, 3rd edition, Lewis Publications, 1997.

[12] Wikipedia the free Encyclopedia, June 2006.

[13] ElHaggar, M., Elkady, M., Ezzeldeen, S., The Complete Guide in Air Pollution and control Technologies, Dar Elfekr Elarabi, 2003 (in Arabic).

[14] www.masterscieng.sunyab.edu/ethics/index.html

[15] www.masterscieng.sunyab.edu/ethics/abet.html

[16] Paul Wright Introduction to Engineering, $2^{\text {nd }}$ Ed., John Wiley \& Sons 1994.

[17] www.masterscieng.sunyab.edu/ethics/chemi.html 